\title{
IMO 2017: Bronze für den Berliner Branko Juran
}

\author{
Kristina Vaillant
}

\begin{abstract}
Branko Juran hat im Juli 2017 bei der 58. IMO in Rio de Janeiro eine Bronzemedaille errungen - eine von vier Medaillen, die das sechsköpfige Team aus Deutschland insgesamt gewann. Gemeinsam mit vier Kollegen aus dem deutschen Team wird er ab dem Wintersemester 2017 an der Uni Bonn Mathematik studieren.
\end{abstract}

\begin{abstract}
Herr Juran, herzlichen Glückwunsch, Sie haben eine Bronzemedaille aus Brasilien mitgebracht! Wie haben Sie die Mathematik-Olympiade erlebt?
\end{abstract}

Die Atmosphäre war eigentlich sehr schön, es ist ja ein total multinationaler Wettbewerb. 615 Schüler aus 111 Ländern. Ja, und ziemlich cool war, dass es einen Aufenthaltsraum gab, wo man Tischkicker spielen konnte. Da ist man mit den anderen Mannschaften ins Gespräch gekommen.

\section{Haben Sie besondere Begegnungen erlebt?}

Ich habe ständig Leute aus Ländern getroffen, aus denen ich vorher noch nie jemandem begegnet war. Wir haben viel mit dem israelischen Team unternommen, das war letztes Jahr bei der Mathe-Olympiade in Hongkong auch schon so. Auch mit den Teilnehmern des britischen Teams haben wir uns viel unterhalten und mit einem Teilnehmer aus Syrien, das war spannend.

Der Sohn des syrischen Präsidenten, Hafez al-Assad, war auch dabei.

Ja, aber den habe ich nur einmal gesehen, gesprochen habe ich nicht mit ihm. Seine Teilnahme war in Deutschland ein Riesenthema in den Medien, in Rio hat das niemanden interessiert. Wir haben überhaupt erst durch die deutsche Presse davon erfahren.

Kommen wir zu den Klausuren. Die fanden nach der feierlichen Eröffnung mit der Parade der Teams am dritten und vierten Tag der Olympiade statt. Sie haben bei den Klausuren zwei der insgesamt sechs Aufgaben komplett gelöst und eine dritte teilweise. Eine der sechs Aufgaben galt als besonders schwer.

Das war Aufgabe 3, und das war die schwierigste Aufgabe aller Zeiten bei der IMO. Nur sieben Teilnehmer haben überhaupt Punkte für diese Aufgabe bekommen, zwei davon die volle Punktzahl. Der eine hat dadurch Gold gewonnen, der andere eine Silbermedaille. Aus unserem Team haben sich zwei daran probiert und null Punkte bekommen. Ich habe die Aufgabe gar nicht erst angefangen.

Worum ging es bei der Aufgabe?
Eigentlich ist sie gar nicht so schwer zu erklären. Ein Hase rennt vor einem Jäger davon. Beide starten am selben Punkt. Der Hase ist für den Jäger unsichtbar, aber der Jäger hat ein Ortungsgerät. Das Gerät zeigt den Punkt an, an dem sich der Hase aufhält, aber nicht genau. Der Jäger weiß also nur, dass sich der Hase im Umkreis von einem Meter um den Punkt aufhält. Jedes Mal wenn der Hase einen Meter weiterläuft, gibt er ein Signal ab und der Jäger muss hinterherlaufen. Aber der kennt die Richtung nur ungefähr. Nun war die Frage, ob der Hase es mit irgendeiner Taktik schaffen kann, nach einer Milliarde Schritte mindestens 1 oo Meter zu entkommen. Das ist eine absolut ungewöhnliche Aufgabe.

\section{Wo lag für Sie die Schwierigkeit?}

Für eine solche Aufgabe gibt es kein Standard-Lösungsverfahren, das muss man selbst rauskriegen. Das eine Problem war also, dass man so eine Aufgabenstellung nicht trainieren kann. Das andere Problem, dass die Aufgabe offen formuliert war: Kann der Hase entkommen? Es gab zwei Wege, die Frage zu beantworten: Entweder man findet eine Strategie für den Jäger, damit er den Hasen einholt, oder für den Hasen, damit er entkommen kann. Normalerweise ist bei solchen offenen Fragen schnell klar, welche Richtung die richtige ist, weil eine irgendwie zu leicht oder zu abstrus wäre. Aber hier war für mich nicht klar erkennbar, welchen Lösungsweg man verfolgen sollte. Man brauchte viel Fingerspitzengefühl und ein bisschen Glück, um die richtige Richtung zu erahnen. Und einer aus unserem Team hat eben genau die falsche Richtung eingeschlagen. Der hat gedacht, okay, der Jäger schafft es irgendwie immer den Hasen einzuholen, hat das dann ein paar Stunden durchexerziert und am Ende festgestellt, das klappt nicht. Aber das sind dann trotzdem null Punkte.

Zu Beginn der Klausuren gab es eine Fragerunde zu den Aufgaben. War das hilfreich?

Man kann Fragen stellen, aber eigentlich nur Verständnisfragen. Dieses Jahr habe ich das tatsächlich gemacht, weil mir eine Formulierung nicht ganz klar war. Aber man bekommt da keine große Hilfe. Manchmal lautet die Antwort einfach: Bitte lesen Sie die Fragestellung erneut. 
